\title{
An Opinion Survey of Reported Benefits From the Use of Stereolithographic Models
}

\author{
Douglas M. Erickson, DDS, MS, * \\ David Chance, DDS, MS, + \\ Stepben Schmitt, DDS, MS, $\neq$ and James Matbis $\mathfrak{l}$
}

\begin{abstract}
Purpose: The aim of the study was to determine the possible benefits from 3-dimensional epoxy stereolithographic (SL) models produced from computed tomography (CT) and magnetic resonance imaging (MRI) data.
\end{abstract}

Materials and Methods: Surgeon's opinions about the use of SL models for diagnosis, treatment planning, practicing surgery preoperatively, within the operating room during surgical procedures, and for the construction of custom titanium implants or surgical devices were tabulated, using a 2-page survey.

Results: Most of the surgeons found SL models useful in all phases of planning and implementation of surgical procedures. Sixty-five percent found that exposure to the SL model changed the way in which they approached the patient's surgery. They also determined that there was a median timesavings of $20 \%$ in expended operating room and anesthesia time. Sixty-two percent of surgeons believed the models were important for proper diagnosis.

Conclusions: Most surgeons using 3-dimensional epoxy SL models for surgical procedures found them beneficial for diagnosis, treatment planning, as a reference during surgery, and in the fabrication of custom implants and surgical devices that afforded surgical solutions previously not available. Patients were believed to have received better care, because the surgeons had more knowledge of their unique anatomy before surgery. Through the use of these models, the patients experienced shorter surgical procedures, with more predictable results.

The stereolithography (SL) process is a rapid prototyping method that allows the fabrication of anatomically accurate 3-dimensional (3D) epoxy and acrylic resin models from various types of data. A myriad of data sources can be assimilated, including computed tomography $(\mathrm{C} T)$, magnetic resonance imaging, and laser scanning.

Clinical experience has found many advantages in the use of $3 \mathrm{D}$ models. These models enhance the

"LtCol, USAF; Chief, Dental Laboratory Services, Wilford Hall Medical Center, Lackland $\Lambda F B$, San $\Lambda$ ntonio, TX.

tCol USAF; Project Manager, Defense Dental Standards Application, Washington, DC.

$\ddagger$ Col(Ret) USAF; Chairman, Department of Prosthodontics, Wilford Hall Medical Center, Lackland AFB, San Antonio, TX.

\$Stereolithography Laboratory Technician, Wilford Hall Medical Center, Lackland, AFB, San Antonio, TX.

Address correspondence and reprint requests to LtCol Erickson: 1212 Medical Arts Building, 324 W Superior St, Duluth, MN 55802; e-mail: dmerickson@compuserve.com

This is a US government work. There are no restrictions on its use. 0278-2391/99/5709-0003\$0.00/0 ability to visualize patient's unique structures before surgery. This aids in the planning of surgery and other treatments. The surgeons also can rehearse proposed surgery on critical structures before committing to the procedure. In the case of craniofacial anomalies, dysplasias, and developmental defects, SL models are particularly useful for designing incisions and surgical resections.

Unsolicited comments from various surgeons have implied that operating room time is reduced with the preoperative modeling of bone graft templates. ${ }^{1}$ These templates can be used during surgery to minimize the trial and error associated with shaping of graft materials. Further timesavings are found with the shaping of prefabricated bone plates before surgery or with use of custom titanium devices (ie, titanium distraction osteogenesis devices, custom temporomandibular joints, fixation devices, and custom implants). Through use of such custom devices, additional treatment options have become available to patients who previously were very difficult or impossible to reconstruct.

The semitransparent models arc a tremendous adjunct in the visualization of body cavities (marrow spaces, craniofacial sinuses, large blood vessels, etc). 
Where there is a perceptible difference in the radiodensities of various body tissues, two-color SL models can be generated to illustrate these differences; for instance, between brain tissue and calvarium, nerves and the surrounding bone, cartilaginous discs and vertebrae, and tumors and the surrounding unaffected tissues.

Epoxy resins used in SL are optically active and are useful in optical stress analysis and photoelastimetry studies. Several of the resins used in SL are also investment castible. The properties of epoxy SL models and the accuracy of this technique lends itself to the fabrication of prosthetic patterns and cast custom devices.

Finally, SL models aid in dental implant design and placement by allowing surgeons to place implant replicas in SL models and to analyze the placement of implants in structures previously not easily accessible to surgeons, such as the pterygoid plates.

The accuracy of the SL machines has improved each year. Currently, 3D Systems, Inc (Valencia, CA), is

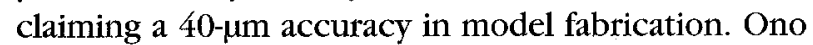

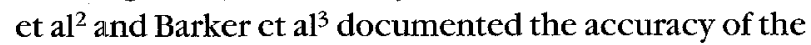
models when using axial and helical CT scans. ${ }^{2,3}$ Taylor et $\mathbf{a l}^{4}$ went a step further, comparing the axial with the helical CT scanning protocols, showing that the axial scans produced a more accurate model than the helical scans and that the integral dose of radiation was statistically the same for the axial and helical scan protocols.

The purpose of this study was to subjectively assess the role of SL in decreasing operating room time improving patient outcomes, and in cost-savings.

\section{Materials and Methods}

The stereolithography apparatus used to fabricate the models was the SLA-500 manufactured by 3D Systems, Inc. This system of rapid prototyping builds models by segmenting a digital representation of the model into many small cross sections and tracing each segment sequentually with an Argon ion laser onto the surface of a vat of liquid epoxy photopolymer. The epoxy becomes selectively hardened where the laser exposes the photopolymer. Once a cross section has been generated, the platform supporting the model drops down an increment into the vat of photopolymer and the laser traces the next cross section, bonding it to the first. The process continues until the entire model has been generated; usually 18 to 48 hours, depending on the part size, complexity, and the number of models being generated at one time. At the completion of the model fabrication, it emerges from the photopolymer $80 \%$ polymerized. After cleaning, the polymerization process is completed in an ultraviolet light chamber.
CT scans were the primary source of data for this study. These scans provided both axial $1 \times 1-\mathrm{mm}$ data and $3-\mathrm{mm}$ helical data reconstituted to $1-\mathrm{mm}$ slices. The data were manipulated with the MIMICS software written by Materialise, Inc, Belgium, and run on Silicon Graphics Indigo Extreme, Hewlett-Packard XU workstations, and a Hewlett-Packard NetServer E30.

Surgeons participating in the study were located at the Wilford Hall Medical Center, San Antonio, TX; Brooke Army Medical Center, San Antonio, TX; David Grant Medical Center, Vacaville, CA; the University of Texas Health Sciences Center, San Antonio, TX; and Tripler Army Hospital, Honolulu, HI. SL models also were fabricated for surgeons from the departments of Plastics and Reconstructive Surgery, Otolaryngology, Neurosurgery, Oral and Maxillofacial Surgery, Pediatrics, Orthopaedics, Prosthodontics, and Periodontics. Cases included a wide variety of procedures such as the separation of Siamese twins, reconstruction of congenital orbital hypotelorism, the diagnosis and treatment of numerous craniofacial clefts, treatment of traumatic gunshot wounds, temporomandibular reconstruction, and orthognathic surgery. Delivery of each model was accompanied by a survey and the surgeon was asked to fill out the survey and return it to the Stereolithography Laboratory at the Wilford Hall Medical Center (Fig 1).

\section{Results}

Seventy-six SL models were generated for surgeons during the study period. Thirty-eight of the surveys were returned. Of those returned, $92 \%$ of the models were used for patient care.

During the planning stages, $69 \%$ of the responding doctors used the SL models to diagnose the patient and $62 \%$ of them thought the models were important for a proper diagnosis. Surgeons also believed that the models helped them to improve the planning process for the intended procedure $92 \%$ of the time. Seventythree percent of the surgeons used the models to educate their patients about the proposed surgical procedure. Thirty-eight percent found the models were useful in practicing the procedure preoperatively.

Intraoperatively, $77 \%$ of the reporting surgeons believed that using the models saved operating room (OR) time. Timesavings ranged from $17 \%$ to $60 \%$, with a median timesavings of $20 \%$. Savings in patient anesthesia exposure were similar to the savings found in OR time. Seventy-seven percent of the surgeons thought they saved anesthesia time (median time savings of $20 \%$; range, $16 \%$ to $60 \%$ ). Thirty-eight percent of the surgeons believed that the use of the models minimized wound size and exposure. Seventythree percent of the surgeons referred to the models 


Model Number:
Surgery Date: - Dx: Doctor Name:
1. Did you use this model for patient care? Yes No (circle answer)
If no, why was the model not used?

Note: If you did use the model for patient care, complete the rest of the survey

2. In your opinion, did the stereolithography model save operating room time? Yes No (circle answer)

If yes, by approximately what percentage?

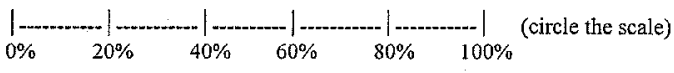

3. Did the patient experience less wound exposure (smaller incisions, faster closure) hecause of this mndel? Yes No (circle answer) If yes, in what way?

FIGURE 1. Stereolithography Tracking Survey.

during the operation to help visualize the approach. Surgeons found that exposure to the SL model changed the way in which they handled the patient's surgery $65 \%$ of the time. Forty-six percent of the doctors thought they were able to offer more complex treatment, or treatment not previously available, because of the use of the models. Previously unavailable procedures included custom temporomandibular joints, intraoral custom titanium distraction osteogenesis devices, and custom titanium implants to replace missing craniofacial bony architecture. Although only $15 \%$ found the SL models essential for care, they found the SL models to be a useful adjunct to patient care $96 \%$ of the time.

\section{Discussion}

The survey results are based on subjective opinions rather than objective data. In several of the categories in which the percentages reported were low-wound size/exposure, preoperative surgical practice, and essential for care-the doctors noted that this was not unexpected because most of the surgical techniques used were previously performed without SL models. Despite this, the surgeons appeared impressed with capabilities and usefulness of the SL system. The respondents believed that the models were useful for the education of patients and surgical residents, regardless of whether the patient had surgery.

Some of the most telling benefits were the reported savings in OR and anesthesia time. Using the $20 \%$ median value, the $\mathrm{SL}$ model would enable surgeons to save $3 \frac{1}{2}$ hours of time during an 18 -hour orthognathic surgery. In traumatized mandibles, the surgeons stated that bending the surgical reconstruction plate for a mandibular reconstruction could take 1 to 2 hours of trial and error intraoperatively. By bending fixation devices against a SL model preoperatively, the surgeons frequently saved 1 to 2 hours of OR time. ${ }^{1}$ Use of SL models for shortening surgcry could hypothctically minimize additional surgical trauma, blood loss, risk of infection, and postoperative complications. Also, by saving operating room time, it is possible to schedule more cases per day, resulting in increased productivity and decreased patient costs.

Another benefit reported included the ability to know the extent of orthognathic movements possible with greater precision than with traditional model surgery prediction techniques. With SL models, and using the Rahn Model Block, surgeons were able to precisely determine the proposed surgical movement of bony segments in a way not possible with the traditional stone casts mounted on an articulator.

Acrylic resin templates also can be fabricated preoperatively to aid in the creation of ideally shaped donor bone. These templates can be fabricated against the SL 
model to determine, with great precision, the exact shape and volume of graft material necessary for an intended surgery, thus minimizing the amount of graft material harvested and the surgical trauma done to a secondary donor site. The acrylic templates enhanced the surgeon's ability to visualize the patient's bony structures contralateral to the surgery site without soft tissue interference, improving the ability to achieve true bony and soft tissue symmetry.

Another benefit described by surgeons was that intraoral and extraoral implants were more easily placed because the exact location of the sinuses, nerves, and bony concavities could be visualized through the semitransparent models. In 1 case, an endosseous implant was placed in the pterygoid plate to achieve vertical support for an obturator after the patient lost most of his maxilla due to a tumor excision.

Finally, in several instances, the use of SL models eliminated second surgeries. Because more is understood about the patient's anatomy, it is possible to reconstruct a patient's anatomy during the same procedure in which tumor ablation is performed with devices fabricated preoperatively.

The impact on specific procedures should be studied to objectively document the value of these models. Some procedures are more likely to benefit than others. With specific findings, actual cost savings can be documented. Included within those savings should be those from decreased postoperative complications.
There is little doubt that the surgeons surveyed found the models very beneficial, even with procedures previously accomplished with other time-tested techniques. They found the SL models enhanced their ability to properly diagnose and treatment plan. In a few cases, surgical solutions were found for patients which, previous to the 3D models, had no surgical options. Surgeons also found the models were helpful in the education of patients and surgery residents. As use of SL models becomes more common, studies must be completed to objectively document the cost savings so that insurance companies will consider payment for them. Patients are the ultimate beneficiaries of this technology; they receive better care and are treated more efficiently.

\section{References}

1. James WJ, Slabberkuorn MA, Edgin WA, el al: Correction of congenital malar hypoplasia using stereolithography for presurgical planning. J Oral Maxillofac Surg 56:512, 1998

2. Ono I, Hironori G, Kazuyoshi S, et al: Integrated life-sized solid model of bone and soft tissue: Applications for cleft lip and palate infants. Plast Reconstr Surg 93:1363, 1994

3. Barker TM, Earwaker WJ, Lisle DA: Accuracy of stereolithographic models of human anatomy. Aust Radiol 38:106, 1994

4. Taylor JS, Norling BK, Alder ME, et al: Influence of computerized tomography parameters on stereolithographic model quality. J Dent Res 77:198, 1998

5. Imai K, Tsujiguchi C, Toda C, et al: Assessment of simulated surgery for craniosysnostosis using 3-D solid models in terms of operating time and blood loss. Poster presentation, presented at the Meeting of the International Society of Craniofacial Surgery, Santa Fe, CA, 1997 